Benchmarks

\section{YGFP: a spectral variant of GFP}

\author{
Flemming G. Hansen ${ }^{1}$ and Tove Atlung ${ }^{2}$ \\ ${ }^{1}$ Center for Systems Microbiology, Department of Systems Biology, Technical \\ University of Denmark, Lyngby, Denmark and ${ }^{2}$ Department of Science, \\ Systems, and Models, Roskilde University, Roskilde, Denmark
}

BioTechniques 50:411-412 (June 2011) doi 10.2144/000113691

Keywords: Fluorescence microscopy; fluorescent spectra; photo-bleaching; fluorescent ParB foci; multifluorescence cell labeling; mOrange

Supplementary material for this article is available at www.BioTechniques.com/article/113691.

We describe YGFP, a slow bleaching green fluorescent protein (GFP) with unique spectral properties. YGFP is derived from an Escherichia coli codon-optimized synthetic $g f p$ mutant 2 derivative. In addition to the GFP-mut 2 changes, it also carries S202F and T203I substitutions. YGFP can be used as a substitute for yellow fluorescent protein (YFP) in experiments in which two or more fluorescent proteins are fused to different cellular protein components, expanding the ability to study multiple labeled proteins in a cell at once.

The green fluorescent protein (GFP) of the jellyfish Aequorea victoria has been used extensively in cell biology studies within the last 15 years, including studies of bacterial gene expression and protein localization using GFP-protein fusions. The needs of these studies prompted changes to the original GFP sequence to improve stability, spectral characteristics, and folding properties (for a review, see Reference 1 ).

The codon usage of the original GFP gene created problems when trying to express the protein in other organisms (2). To prevent similar problems in Escherichia coli, we synthesized a $g f p$ gene optimized for use in this organism. This synthetic $g f p$ gene uses codons similar to ribosomal and other abundant proteins in E. coli (see Supplementary Materials for details). The gene carries the GFP mutant 2 changesS65A, V68L, S72A - in the chromophore (3) as well as a D234 H change to provide a restriction site in the 3 '-end. Our final GFP (GFP-mut2) also carries an accidental M153V alteration, which increased fluorescence (see Supplementary Figure S4). The DNA sequence of plasmid pFH2191 (Supplementary Figure S1) carrying our synthetic $g f p$ gene is deposited in GenBank (account no. AF325903). spectra). All combinations except those carrying the S202F-T203I or V163A changes had spectral properties similar to mutant 2 (3).GFP with the V163A mutation displayed a red shift (plus 5 $\mathrm{nm}$ excitation; plus $2 \mathrm{~nm}$ emission). All combinations carrying the S202F-T203I change exhibited excitation and emission spectra that were very different from the GFP-mut 2 spectra (Figure 1 and Supplementary Figure $S 4$ ).

We selected the S202F-T203I along with M153T and V163A for inclusion in our new fluorescent protein, which we named YGFP. The excitation spectrum of YGFP is similar to that of yellow fluorescent protein (YFP), while the emission maximum is very close to that of GFP. Figure 1 shows the spectra of GFP-mut2, YGFP, YFP (equal to our GFP-mut2 except for the T203Y change (7)), and a cyan fluorescent protein (CFP) synthesized from the E. coli codon optimized and modified $c f p$ gene.

Surprisingly, all the combinations carrying the S202F-T203I changes (see Supplementary Figure S4) were not excited at $400 \mathrm{~nm}$, which is the wavelength reported to excite GFP carrying these two mutations (6). We replaced the chromophore of one of the plasmids (pFH2509) with a wild-type chromophore and found that the spectral characteristics became similar or identical to those published by Heim et al. (6) (excitation at $400 \mathrm{~nm}$, no excitation at $480 \mathrm{~nm}$, and an emission maximum at $\sim 510 \mathrm{~nm}$; data not shown). At this point, we realized that the spectral properties of the YGFP were virtually identical to those of the GFP mutant 3 derivative (3). Supplementary Figure S3 for details and
A

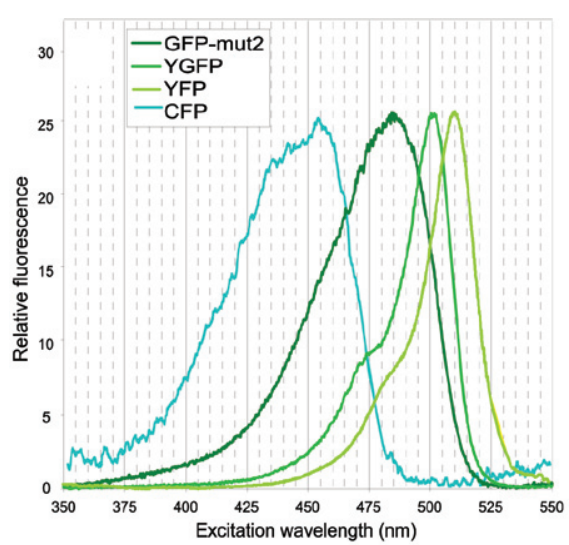

B

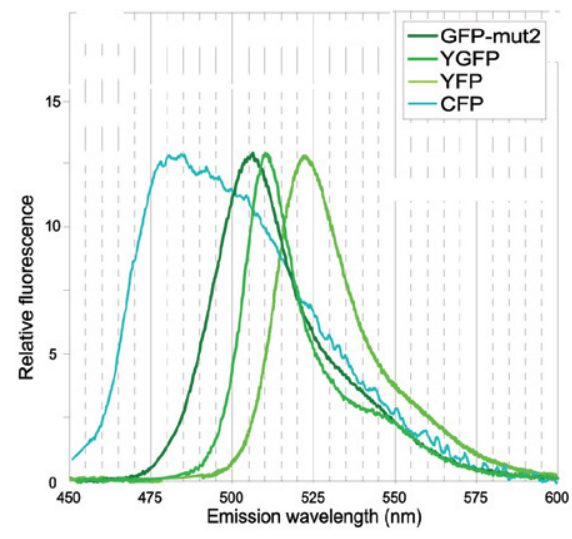

Figure 1. Spectral properties of GFP-mut2, YGFP, YFP, and CFP. Excitation spectra were recorded at emission wavelengths of $508 \mathrm{~nm}$ for GFP and YGFP, $475 \mathrm{~nm}$ for CFP, and $528 \mathrm{~nm}$ for YFP. Emission spectra were obtained using excitation wavelengths of $482 \mathrm{~nm}$ for GFP, YGFP, YFP, and $450 \mathrm{~nm}$ for CFP. CFP is derived from our GFP-mut2 with the following amino acid changes: F64L A65T Y66W L70V. This CFP fluoresces more than the commercially available enhanced CFP (ECFP), which has an additional N145I change (data not shown). 


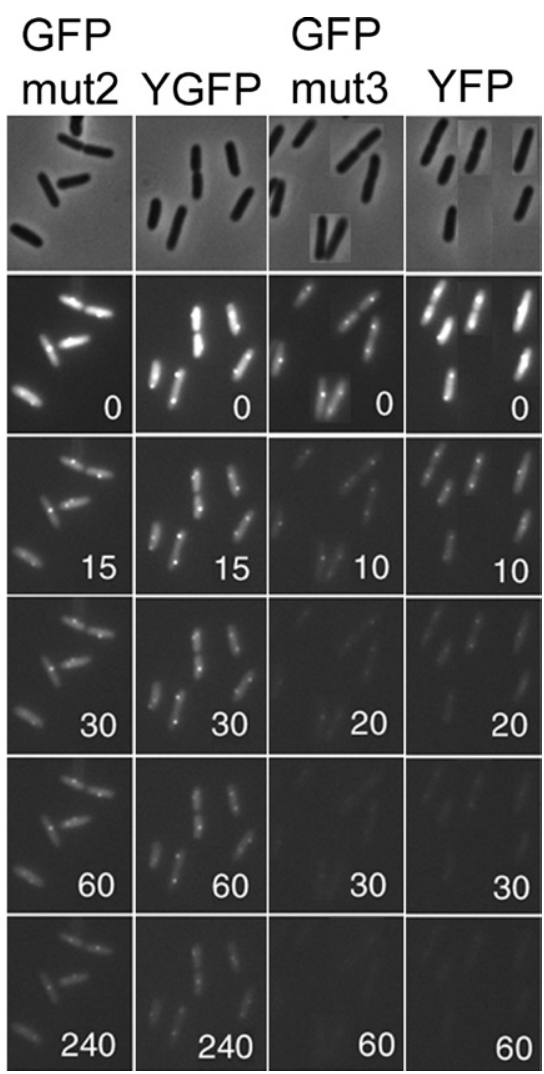

Figure 2. Bleaching properties of fluorescent proteins. Photomicrographs of $E$. coli cells with a pMT1 parS sequence inserted at the origin of replication between the asnA and asnC genes. The four strains carried four different plasmids with the genes for GFP-mut2, YGFP, GFP-mut3, and YFPs fused to the pMT1 parB gene (see Supplementary Figure S4 for plasmid structure). All genes were $E$. coli codon-optimized and controlled by an arabinose promoter. Cells in logarithmic growth were induced with $0.2 \%$ arabinose for 30 min and kept on ice until pictures were taken. At this induction level, excess fluorescent fusion protein is synthesized and binds nonspecifically to the nucleoid in addition to the specific binding to the parS sequence in the origin. All pictures are 0.99-s exposures of bacteria excited for the periods indicated in seconds on the individual pictures.

To examine functionality of the new fluorophore, YGFP and CFP were fused to different ParB partition proteins from bacteriophage P1 and plasmid pMT1, respectively. These two fusion proteins were used to successfully fluorescently label $\operatorname{par} S$ sequences at different positions on the chromosome (8). With GFP-mut2 and YFP ParB protein fusions, we could also distinguish fluorescent labeling at two different positions on the chromosome in the same cell. However, YFP fluorescence bleached very rapidly. We compared the bleaching properties of fluorescent foci at a $\mathrm{PMT} 1$ par $S$ sequence formed by the
GFP-mut2, the YGFP, the GFP-mut3, and the YFP fusions to the pMT1 ParB protein. Figure 2 shows fluorescent GFP-mut2 and YGFP foci present after 240 s of exposure, whereas GFP-mut 3 and YFP foci disappear very rapidly, becoming difficult to observe after $30 \mathrm{~s}$ and virtually absent after $60 \mathrm{~s}$.

YGFP and YFP have very similar $\mathrm{pH}$ sensitivities (9), but YGFP is much less halide-sensitive than YFP (10). Halide and $\mathrm{pH}$ sensitivities of GFP-mut2 and YGFPs are shown in Supplementary Figure S6.

We conclude that YGFP is a potentially advantageous fluorescent reporter protein because it is more photo-stable than GFP-mut3 (3) and YFP; the spectral properties of YGFP make it very useful for polyfluorescence labeling and visualization of different cellular positions; and it is not excited at wavelengths $\geq 525 \mathrm{~nm}$, so it can be used with mOrange fluorescent protein (11) using single band pass filter sets for both proteins (e.g., Semrock GFP-3035C for YGFP and SpGold-B for mOrange), which are also compatible with a filter set for mCherry (Semrock LF594/LP-A). In conclusion, it should be possible to simultaneously record the fluorescent proteins CFP, YGFP, mOrange, and mCherry (11), allowing visualization of four different fluorescent protein fusions in the same cell.

\section{Acknowled gments}

We thank Søs Koefoed for expert technical assistance and many of our colleagues for discussing our fluorescent proteins. The spectrofluorimeter we used in this study was a gift from the Carlsberg Foundation. The project was supported by grants from the Danish Natural Science Research Council.

\section{Competing interests}

The authors declare no competing interests.

\section{References}

1. Tsien, R.Y. 1998. The green fluorescent protein. Annu. Rev. Biochem. 67:509-544.

2. Cormack, B.P., G. Bertram, M. Egerton, N.A. Gow, S. Falkow, and A.J. Brown. 1997. Yeast-enhanced green fluorescent protein (yEGFP)a reporter of gene expression in Candida albicans. Microbiology 143:303311.

3. Cormack, B.P., R.H. Valdivia, and S. Falkow. 1996. FACS-optimized mutants of the green fluorescent protein (GFP). Gene 173:33-38.
4. Crameri, A., E.A. Whitehorn, E. Tate, and W.P. Stemmer. 1996. Improved green fluorescent protein by molecular evolution using DNA shuffling. Nat. Biotechnol. 14:315319.

5. Siemering, K.R., R. Golbik, R. Sever, and J. Haseloff. 1996. Mutations that suppress the thermosensitivity of green fluorescent protein. Curr. Biol. 6:1653-1663

6. Heim, R., D.C. Prasher, and R.Y. Tsien. 1994 Wavelength mutations and posttranslationa autoxidation of green fluorescent protein. Proc. Natl. Acad. Sci. USA 91:12501-12504.

7. Østergaard, H., A. Henriksen, F.G. Hansen, and J.R. Winther. 2001. Shedding light on disulfide bond formation: engineering a redox switch in green fluorescent protein. EMBO J. 20:5853-5862.

8. Nielsen, H.J., J.R. Ottesen, B. Youngren, S.J. Austin, and F.G. Hansen. 2006. The Escherichia coli chromosome is organized with the left and right chromosome arms in separate cell halves. Mol. Microbiol. 62:331-338.

9. Jayaraman, S., P. Haggie, R.M. Wachter, S.J. Remington, and A.S. Verkman. 2000 Mechanism and cellular applications of a green fluorescent protein-based halide sensor. J. Biol. Chem. 275:6047-6050.

10. Wachter, R.M. and S.J. Remington. 1999. Sensitivity of the yellow variant of green fluorescent protein to halides and nitrate. Curr. Biol. 9:R628-R629.

11. Shaner, N.C., R.E. Campbell, P.A. Steinbach, B.N. Giepmans, A.E. Palmer, and R.Y. Tsien. 2004. Improved monomeric red, orange and yellow fluorescent proteins derived from Discosoma sp. red fluorescent protein. Nat. Biotechnol. 22:1567-1572.

Received 2 November 2010; accepted 3 May 2011.

Address correspondence to Tove Atlung, Department of Science, Systems, and Models, Roskilde University, Building 18.1, DK-4000 Roskilde, Denmark. e-mail: atlung@ruc.dk.

To purchase reprints of this article, contact: biotechniques@fosterprinting.com 